\section{CARMEN DE BURGOS, LA APM Y AQUELLAS ADMIRABLES CHICAS DEL 98}

\author{
Bernardino M. Hernando \\ Universidad Complutense de Madrid \\ Asociación de la Prensa de Madrid \\ Junta Directiva \\ Calle de Juan Bravo, 6 \\ 28006, Madrid \\ 915850010 \\ bmhernando@apmadrid.es
}

\begin{abstract}
It enters the five women journalists pertaining to the Association of the Press of Madrid (APM) was the writer, professor, lecturer and hardened travelling Carmen de Burgos, Colombine call. It and the other four, Jesusa Granda, participant in the foundation of the APM in 1895, Atocha Ossorio and Gallardo, Salomé Núñez de Topete and Consuelo Álvarez Pool trained a made an effort group of admirable women fighters by the culture and the human rights. Identified with the advanced to "generation of 98" if this nonhuibera be as chauvinistic as was almost everything then.
\end{abstract}

KEY WORDS: Press Association of Madrid; Carmen de Burgos; Generation of '98; spanish press; feminism; women journalists.

La Asociación de la Prensa de Madrid (APM) se fundó en 1895. De las 173 personas consideradas "socios fundadores", sólo una era mujer: Jesusa Granda y Lahín, con el número 67. Y desde la APM, aun hoy, esgrimimos el dato como un triunfo. No hace falta decir por qué. Además tampoco la APM empezó distinguiéndose por su apoyo a la mujer periodista. Tardó mucho en aceptarla entre sus asociados con naturalidad. Tuvo una mujer fundadora, es verdad, pero la segunda mujer que quiso ser de la APM, Atocha Ossorio y Gallardo, ya entrado el siglo XX, hubo de sufrir rechazos y suspicacias'.

Carmen de Burgos ingresa en la asociación, en 1907, el mismo año en el que muere Jesusa Granda. Ingresa con el número 1.030 y es la quinta mujer asociada. Cinco mujeres en 12 años no es mucho. 0 sí, según se mire.

Sobre todo si se tiene en cuenta la calidad de estas cinco mujeres pioneras en el periodismo madrileño. Las cinco ocuparian un lugar en aquel grupo literario y periodístico

\section{CARMEN DE BURGOS, THE APM AND THOSE ASTONISHING GIRLS OF THE 98}

RESUMEN: Entre las cinco primeras mujeres periodistas pertenecientes a la Asociación de la Prensa de Madrid (APM) estaba la escritora, profesora, conferenciante y empedernida viajera Carmen de Burgos, llamada Colombine. Ella y las otras cuatro, Jesusa Granda, participante en la fundación de la APM en 1895, Atocha Ossorio y Gallardo, Salomé Núñez de Topete y Consuelo Álvarez Pool formaban un grupo esforzado de admirables mujeres luchadoras por la cultura y los derechos humanos. Identificadas con los ideales sociales más avanzados podrian haber pertenecido a la "generación del 98" si esta no hubiera sido tan machista como era casi todo en aquella época.

PALABRAS CLAVE: Asociación de la Prensa de Madrid; Carmen de Burgos; Generación del 98; prensa española; feminismo.

que se dio en llamar "generación del 98" si ésta no hubiera estado formada exclusivamente por sesudos varones. Ninguna mujer tuvo opción a ser considerada parte del grupo (que, como hoy sabemos, no lo fue tanto) a pesar de haber compartido con él ideas e ilusiones, actividades y sinsabores.

María Atocha Ossorio y Gallardo (ingresó en APM, 1906), Salomé Núñez de Topete (en APM, 1906) y Consuelo Álvarez Pool (APM,1907) son, con Jesusa Granda y Carmen de Burgos, aquellas admirables chicas del 98. Las cinco periodistas y asociadas a la APM, lo que, por entonces, tenía ciertos visos de arriesgada avanzadilla. La APM no era el Ateneo ni una asociación política o sindical, ni un club de intelectuales. Era, apenas, una sociedad de socorros mutuos pensada para servir de ayuda a los pobres periodistas, casi siempre vecinos de la miseria. Sin embargo, la mera pertenencia a ella significaba una especie de batalla ganada, de grito de presencia y solidaridad. Y todo eso, siendo mujer en una sociedad tan machista (incluida la 
APM), equivalía a estar en la peligrosa vanguardia de una profesión casi tan misteriosamente reservada a los varones como la Academia o el Congreso. Ser mujer y ser periodista y asociada a la APM, a finales del siglo XIX y bien entrado el XX, era una clase de heroísmo.

Estas cinco mujeres coetáneas ${ }^{2}$ no formaron pandilla, no eran todas contertulias, pero todas se conocieron, com-

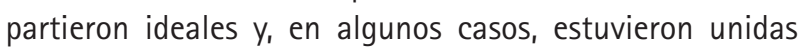
por la amistad. Lo que basta para tratarlas como "aquellas admirables chicas del $98 "$.

Por supuesto que hubo otras muchas mujeres, incluso de mayor relieve social e intelectual, pero aqui solo queremos hablar de estas como pioneras periodisticas de la APM.

\section{LOS POCOS ESPACIOS ABIERTOS}

En el Madrid de finales del XIX, apenas hay lugar para las mujeres inquietas, luchadoras y con ganas y talento para participar en la vida cultural o política. Aparte de grupos e instituciones amistosas $y$, por supuesto privadas, a veces casi clandestinas, entre los pocos espacios públicos accesibles a las mujeres estaban el Ateneo, la Escuela Normal, los periódicos y, sorprendentemente, el Cuerpo de Telégrafos. En ellos, la mujer con talento y ganas podía ejercer sus dotes intelectuales sin tener que pedir perdón. Aunque, faltaría más, con todos los condicionantes inevitables de la época. Uno de ellos, el frecuente uso de seudónimos que sonaban, a veces, como un guiño de complicidad y disimulo: la Núñez Topete, Melita; Consuelo Álvarez, Violeta; Carmen de Burgos, Colombine y otros varios; Atocha Ossorio Gallardo, Atocha o María Atocha...

En estos cuatro espacios, completados por la creación literaria que, desde tiempo inmemorial, produjo escritoras importantes, actuaron nuestras cinco pioneras.

En el Ateneo se movieron todas con soltura y eficacia, sobre todo Melita, Violeta y Colombine. En la Escuela Normal ejercen y destacan Jesusa Granda y Carmen de Burgos. Jesusa es nombrada profesora de Pedagogía Gimnástica en la recién fundada Escuela Central de Gimnástica (1886); y Carmen obtiene plaza como profesora numeraria de la Normal de Maestras de Guadalajara en
1901, para pasar a la de Toledo en 1907, y a la Normal de Madrid en 1911.

Todas colaboraron en varias publicaciones periódicas, pero los periódicos diarios en que más coincidieron fueron el castelarista El Globo (Granda, Ossorio, Carmen), el republicano El País (Consuelo...), El Liberal (Salomé...), Heraldo de Madrid (Carmen...). Carmen de Burgos fue la primera periodista española profesional, la primera que firmó un contrato de redactora (Diario Universal, 1903; luego redactora de Heraldo...), la primera corresponsal de guerra (África, 1909)... Sin embargo, nunca se dedicó exclusivamente al periodismo. Salomé Núñez Topete y Atocha Ossorio y GaIlardo tampoco se dedicaron exclusivamente al periodismo pero siguieron a Carmen en cuanto a intensidad y continuidad en sus trabajos periodisticos. Atocha era de familia de periodistas, hija de Manuel Ossorio y Bernard, autor del famoso catálogo de periodistas españoles del siglo XIX, en el que Atocha tuvo importante colaboración y en el que figura ella misma como "redactora única del semanario La Elegancia (1899-1900)"3. Salomé, de la familia de los ilustres militares Topete, estaba lejanamente emparentada con Juan Valera (que se llamaban entre sí "tío" y "sobrina") por el apellido Viaña, segundo apellido del padre de Juan Valera y segundo apellido del abuelo materno de Salomé. Más que semejante lejanisimo parentesco contaba la amistad intima entre Salomé y Carmen Valera, la hija y primera editora de don Juan Valera. Salomé era también muy amiga de Carmen de Burgos a la que ayudó, como entrevistada, en el perfil que Colombine hizo de Valera ${ }^{4}$.

Salomé, por sus obras literarias, sus traducciones y su actividad periodistica, merece una atención que todavía no ha tenido: escribe en El Liberal y Mundo Gráfico y es corresponsal en Madrid de Diario de la Marina (La Habana), por la misma época (1896) en que en Diario de la Marina escribían desde Madrid Núñez de Arce, Andrés Mellado, José Echegaray... Salomé es la única de la que se conserva, en la APM, un expediente de asociada en el que figuran algunos textos manuscritos (una carta a Francos Rodríguez y tarjetas dirigidas al secretario general, Eduardo Palacio-Valdés) así como la constancia de que, en enero de 1929 solicitó y le fue concedida la baja de la APM. Se dio de baja cuando la APM elevó la cuota de asociados a cinco pesetas.

Consuelo Álvarez Pool, Violeta, era igualmente amiga de Carmen de Burgos y tertuliana en los Miércoles de Co- 
lombine, la tertulia literaria en casa de Carmen (1906) que reunía a periodistas y escritores de la talla de Blasco Ibáñez, Salvador Rueda, Cansinos-Asséns, Ramón Gómez de la Serna, José Francés...

Consuelo pertenece al cuerpo de auxiliares de Telégrafos, como Clara Campoamor y tantas otras mujeres que buscaban en aquel trabajo no solo el pan sino también la sal. Acabó siendo un cuerpo de élite femenina. En 1909 $y_{1}$ en segunda tanda, en 1915, fue Director General de Comunicaciones José Francos Rodríguez, médico, político y periodista, directivo de la APM y futuro presidente, segundo presidente, sucesor de Moya.. Consuelo conectó enseguida con Francos Rodríguez y cuando éste estableció, por primera vez, un gabinete de prensa en Telégrafos, Consuelo formó parte de él. Sus colaboraciones en El País, donde había sido también redactor Francos Rodríguez, y en la revista de Telégrafos llamaban la atención por su valentía y coherencia.

\section{LOS PRIMEROS Y MÁS ALTOS CARGOS DE LA APM}

Por desgracia (iy seguimos sin acostumbrarnos a este tipo de desgracias!) y con la excepción ya señalada de Salomé Núñez y Topete, no ha quedado rastro de estas pioneras en los expedientes de asociados de la APM y apenas huella en las actas de las juntas directivas. Sin embargo, su relación con los primeros y principales directivos de la asociación fue cercana y continuada. Alfredo Vicenti, fundador de la APM, y Francos Rodríguez (que lo fue todo en política, menos presidente del gobierno) fueron directores de El Globo. Francos Rodríguez dirigió también Heraldo de Madrid y, como alcalde de la capital, tuvo más de una intervención favorecedora de las ideas y los ideales de este grupo de mujeres. El primer presidente de APM, Miguel Moya, director de El Liberal, fue muy admirado y querido de Carmen de Burgos. Las relaciones de Carmen con Miguel Moya fueron siempre muy cordiales, con alguna ligera nube ${ }^{5}$ que no influyó para nada en el apoyo decidido y valiente que Carmen prestó a Moya cuando este, ya presidente de la Sociedad Editorial de España, el famoso "Trust", que agrupaba a El Liberal, El Imparcial y Heraldo de Madrid, hubo de sufrir el feroz acoso de los partidarios de la huelga de periodistas de diciembre de 1919. Carmen y Ramón Gómez de la Serna, cuya relación amorosa ya era de larga data, apoyaron a Moya con toda su fuerza y sinceridad y de ello dejó constancia Ramón en el capítulo XLVI de su Automoribundia.

Carmen de Burgos muere el 9 de octubre de 1932 y se nos cuenta, en la citada biografía de Concepción Núñez (p. 617) que "pidió la asistencia de su amigo y médico personal Gregorio Marañón". Pero es que, además, Marañón era médico de la APM desde 1911. Y yerno de Miguel Moya (iqué pequeño es el mundo!).

Francos Rodríguez había muerto el 13 de julio de 1931 y, en 1932, el presidente de la APM era Alejandro Lerroux, de buena memoria en la relación con Carmen pues, como político, había apoyado claramente el voto femenino fruto de tantos esfuerzos de Carmen y sus compañeras de lucha.

Estos fueron los dirigentes de la APM que más vinculados estuvieron a estas cinco mujeres, intelectuales y pragmáticas, adoctrinadas entre el espíritu de la llustración y el de la Institución Libre de Enseñanza, en cercanía con el socialismo ideológico y en la vanguardia de los derechos de la mujer. Dejaron huella de sí en la historia del periodismo español. Aunque muchos se hayan empeñado en que fuera una huella en el mar. Es hora de rectificar. 
1 Víctor Olmos, en su minuciosa y magnifica historia de la APM, de la que han aparecido dos volúmenes y está a punto de salir el tercero y último, ha contado la dificultosa elección de Atocha Ossorio y Gallardo (v. tomo I: La Casa de los Periodistas. Asociación de la Prensa de Madrid, 1895-1950, APM, 2006, pp. 157-158).

2 La coetaneidad es relativa y compleja. El baile de fechas, más o menos voluntario, impide muchas veces conocer la edad exacta (iy no solo de las mujeres, como en este caso!). Probablemente, la única de la que hoy puede saberse con exactitud fecha de nacimiento es Carmen de Burgos (1867). Y eso desde no hace mucho tiempo: desde que Concepción Núñez Rey la documentó de forma incontestable en su excelente biografía Carmen de Burgos, Colombine, en la Edad de Plata de la literatura española (Fundación José Manuel Lara, Sevilla, 2005, p. 26). Lo que podemos asegurar, y ello nos basta para nuestro propósito, es que las cinco se conocieron, coincidieron en actividades e instituciones, y murieron en torno a la instauración de la II República (1931-1932), excepto, en ambos extremos, Jesusa Granda que murió en 1907 y Consuelo Álvarez muerta en 1957. Sus ingresos en la APM, con la salvedad de la fundadora Jesusa Granda (1895) coinciden igualmente: en 1906 ingresan Atocha Ossorio y Salomé Núñez Topete, y en 1907 Consuelo Álvarez y Carmen de Burgos. Estas dos últimas, con números seguidos, 1029 y 1030 . No parece aventurado suponer que las cuatro se

Recibido: 14 de mayo de 2010 Aceptado: 7 de junio de 2010
3 En 2004, la Hemeroteca Municipal de Madrid hizo una edición facsímil del Ensayo de un Catálogo de periodistas españoles del siglo XIX, de Manuel Ossorio y Bernard, con motivo del centenario del fallecimiento del autor y fecha de la primera edición del libro (1904).

4 Valera, Juan: Correspondencia. Volumen IV (1884-1887), Castalia, 2005. Edición de Leonardo Romero Tovar (direc.), M. ${ }^{2}$ Ángeles Ezama Gil y Enrique Serrano Asenjo. En pp. 38-39, carta de Valera a Salomé en la que se despide como "tu afmo tío y eterno admirador".

En el libro de Carmen de Burgos Hablando con los descendientes, editado por Compañia Iberoamericana de Publicaciones, Editorial Renacimiento, Madrid 1929 -pp. 183-188- se hace una entrevista a "mi querida amiga, la ilustres escritora Salomé Núñez de Topete, que es intima amiga de la familia de Valera, y hasta creo que algo pariente", en la que Salomé cuenta a Carmen sus recuerdos y conocimientos de Valera.

En el diario $A B C$ de Madrid, edición de la mañana del 27 de enero de 1931, p. 45, en las Noticias Necrológicas y bajo el epígrafe de "Las señoritas de Núñez y Topete" se da cuenta de que "A consecuencia de afecciones pulmonares, y en rápida enfermedad, han fallecido, en la madrugada de ayer, y con sólo unas horas de diferencia, las hermanas señoritas Salomé y Milagros Núñez y Topete, muy estimadas y conocidas en la sociedad madrileña". Se hace luego eco de las actividades periodisticas y literarias de Salomé, de sus colaboraciones en La Nación, de Buenos Aires y Diario de la Mari$n a$, de la Habana y del homenaje que este último periódico había rendido a Salomé con motivo de "sus bodas 
de oro con el periodismo". Lo que nos obligaria a revisar la dada comúnmente como fecha de nacimiento de Salomé, 1871.

5 La "ligera nube" que empañó levemente la relación de Carmen con Miguel Moya fue la negativa de éste a apoyar, desde la APM, la coronación nacional del poeta Salvador Rueda, amigo y contertulio de Carmen a la que dedicó una encendida oda. Es una historia un tanto cómica: Rueda quería ser coronado poeta, a la ma- nera en que lo habian sido Quintana y Zorrilla. "Salvador Rueda anda loco queriendo que lo coronen" dice Ramón en Automoribundia (cap. XXXIII). $Y$ lo coronaron, pero en Albacete, en octubre de 1908, siendo mantenedor de la fiesta literaria (ilo que es la vida!) José Francos Rodríguez, que había sido médico de Hellín, donde se había casado y diputado por Almansa. En 1910 Rueda es coronado igualmente en La Habana. Pero él, y sus amigos, Carmen entre ellos, que- rían que fuera coronado solemnemente en Madrid, y quién sabe si por el mismísimo rey. Quién sabe. Carmen escribió a Moya pidiéndole su apoyo y el de la APM a la que Rueda también pertenecia desde 1897. Por la razón que fuera, vamos a suponer que por mera sensatez, Moya dio la callada por respuesta con disgusto de Carmen y los firmantes de aquella carta de la que da cuenta, en su citada biografia, Concepción Núñez Rey (pp. 187-189). 